\title{
Distributed coordination of self-organizing mechanisms in communication networks
}

\author{
Abdoulaye Tall ${ }^{*}$, Richard Combes ${ }^{\dagger}$, Zwi Altman * and Eitan Altman ${ }^{\ddagger}$ \\ * Orange Labs 38/40 rue du General Leclerc,92794 Issy-les-Moulineaux \\ Email: \{abdoulaye.tall,zwi.altman\}@orange.com \\ ${ }^{\dagger}$ KTH, Royal Institute of Technology, Stockholm, Sweden \\ Email: rcombes@kth.se \\ ${ }^{\ddagger}$ INRIA Sophia Antipolis, 06902 Sophia Antipolis, France \\ Email:eitan.altman@sophia.inria.fr
}

\begin{abstract}
The fast development of the Self-Organizing Network (SON) technology in mobile networks renders the problem of coordinating SON functionalities operating simultaneously critical. SON functionalities can be viewed as control loops that may need to be coordinated to guarantee conflict free operation, to enforce stability of the network and to achieve performance gain. This paper proposes a distributed solution for coordinating SON functionalities. It uses Rosen's concave games framework in conjunction with convex optimization. The SON functionalities are modeled as linear Ordinary Differential Equation (ODE)s. The stability of the system is first evaluated using a basic control theory approach. The coordination solution consists in finding a linear map (called coordination matrix) that stabilizes the system of SON functionalities. It is proven that the solution remains valid in a noisy environment using Stochastic Approximation. A practical example involving three different SON functionalities deployed in Base Stations (BSs) of a Long Term Evolution (LTE) network demonstrates the usefulness of the proposed method.

Keywords-Self-Organizing Networks, Concave Games, SON Coordination, Stochastic Approximation
\end{abstract}

\section{INTRODUCTION}

The Radio Access Networks (RAN) landscape is becoming increasingly complex and heterogeneous with co-existing and co-operating technologies. SON mechanisms have been introduced as a means to manage complexity, to reduce cost of operation, and to enhance performance and profitability of the network. Self-organizing networks enable automation of the configuration of newly deployed network nodes (selfconfiguration), automation of parameter tuning for Key Performance Indicators (KPIs) improvement (self-optimization) and also automation of diagnostic and reparation of faulty network nodes (self-healing). In LTE networks, large scale deployment of the SON functionalities has started with self-configuration SONs to simplify the network deployment, and that of selfoptimization functionalities is expected to follow.

Self-optimization mechanisms can be viewed as control loops, that can be deployed in the management or the control plane. The former is often denoted as centralized SON and

This work has been partially carried out in the framework of the FP7 UniverSelf project under EC Grant agreement 257513 the latter as distributed SON. In the centralized case, the SON algorithms are deployed in the Operation and Maintenance Center (OMC) or in the Network Management System (NMS) which are part of the Operation and Support System (OSS). Centralized SON benefits from abundant data (metrics and KPIs) and computational means necessary for processing and running powerful optimization methods [1], [2]. The main drawback of the centralized approach is related to the long time scale that is typically used, in the order of an hour and more. Hence the SON algorithms cannot adapt the network to traffic variations that occur at short time scales.

The second approach, namely the distributed SON, is more scalable since the optimization is performed locally involving one or several BSs. The main advantage of the distributed SON is its higher reactivity, namely its ability to track quick variations in the propagation conditions or in the traffic [3], [4] and to adapt system parameters in the time scale of seconds (i.e. flow level duration). The higher reactivity sometimes impacts the type of solution sought, namely a solution which targets local minima instead of global minima. However, distributed optimization can also reach global minima [3].

SONs are often designed as stand alone functionalities, and when triggered simultaneously, their interactions are not always predictable. The deployment of multiple control loops raises the questions of conflicts, stability and performance. The topics of conflict resolution, coordination, and a framework for managing multiple SON functionalities are receiving a growing interest (see for example [5]-[7]). Most contributions that have addressed the coordination problem between specific SON functionalities, provide a solution implemented in a centralized [8], [9] or distributed [10] fashion. In a centralized solution, the SON coordination can be treated as a multi-objective optimization [11]. From the standardization point of view, the coordination problem has been addressed as a centralized, management-plane problem [12].

Little material has been reported on distributed, control plane solutions for the coordination problem in spite of its higher reactivity, attractiveness from an architecture point of view and potential performance gain. The aim of this paper is to provide a generic coordination mechanism which is practically implementable. The contributions of the paper are 
the following:

- The problem of SON coordination is analyzed using a control theory/stochastic approximation-based framework.

- The case of fully distributed coordination is addressed.

- It is shown that coordination can be formulated as a convex optimization problem.

- The coordination solution is applied to a use case involving 3 SON functions deployed in several BSs of a wireless network.

A first version of this paper has already been published in [13]. New results presented here include the formulation of the coordination problem as a convex optimization problem with Linear Matrix Inequality (LMI) constraints: stability constraint and connectivity constraints related to the capability of the selforganizing nodes to exchange information via the transport network. The merit of the proposed solution is its capability to handle a large number of control loops and enforce their stability, as illustrated in the use case of SON deployment in a LTE network. To our knowledge, this is the first generic control plane solution to the problem of SON coordination in a mobile network.

The paper is organized as follows: in Section II we state the proposed model for interaction of SON mechanisms running in parallel and the coordination problem to be solved. In Section III we focus on the case where performance indicators are affine functions of the parameters, and propose a practically implementable coordination mechanism. In Section IV we study fully distributed coordination when no exchange of information between SONs is needed, and we show that it is not always possible. In Section $\mathrm{V}$ the coordination problem is formulated as a convex optimization problem which can be fast solved with modern computers. In Section VI we illustrate the application of our model to traffic management in wireless networks with two examples including a use case of the coordination method in a LTE network with three different SON functionalities deployed in each BS. Section VII concludes the paper. In appendices $B$ and $C$ we recall the basic notions of stability for ODEs and linear ODEs respectively.

\section{LIST OF NOTATIONS}

$[\bullet]_{S}^{+} \quad$ Projection on the set $S$

$[A]_{k, k} \quad k^{t h}$ order leading principal submatrix of $A$

$\bar{x} \quad$ Notation for constants often representing target values

$\mathcal{C} \quad$ Set of matrices having the same particular form (e.g., with zero elements at specific positions)

$\dot{\alpha} \quad$ Derivative of $\alpha$ over time

$\mathbb{E}(\bullet) \quad$ Expectation of a random variable

$\mathbb{1}_{\{c o n d\}}(x)$ Indicator function on the set of $x$ values satisfying the condition cond

$\nabla_{x} f \quad$ Gradient of $f$ with regard to $x$

$\operatorname{tr}(\bullet) \quad$ Trace of a matrix

$\theta \quad$ Vector of parameters

$\theta^{*} \quad$ Equilibrium points of system comprizing control loops

$A \prec 0 \quad A$ is negative definite

$A \quad$ Real matrix representing linearized system of SON functions
$A^{T} \quad$ Transpose of matrix $A$

$\operatorname{det}(\bullet)$ Determinant of a matrix

$\operatorname{eig}(A)$ Eigenvalues of $A$

$J F(\bullet)$ Jacobian of $F()$

\section{Problem Description}

\section{A. SON model}

A SON mechanism is an entity which monitors a given performance indicator and controls a scalar parameter. The current value of the performance indicator is observed, and the parameter is modified accordingly to attain some objective. We consider $I>1$ SON mechanisms operating simultaneously. We define $\theta_{i}$ the parameter controlled by the $i$-th SON mechanism and $\theta=\left(\theta_{1}, \ldots, \theta_{I}\right)$ the vector of parameters. The $i$-th SON mechanism monitors a performance indicator $F_{i}(\theta)$ and updates its parameter $\theta_{i}$ proportionally to it. $F(\theta)=\left(F_{1}(\theta), \ldots, F_{I}(\theta)\right)$ is the vector of update of $\theta$.

We say that the $i$-th SON mechanism operates in standalone mode if all parameters but $\theta_{i}$ are kept constant. The $i$-th SON mechanism operating in stand-alone is described by the ODE:

$$
\dot{\theta}_{i}=F_{i}(\theta), \dot{\theta}_{j}=0, j \neq i .
$$

We say that the SON mechanisms operate in parallel mode if all parameters are modified simultaneously, which is described by the ODE:

$$
\dot{\theta}=F(\theta) .
$$

We say that the $i$-th SON mechanism is stable in standalone mode if there exists $\theta_{i}^{*, i}$ for fixed $\theta_{j}, j \neq i$ which is asymptotically stable for (1). The definition of asymptotic stability is recalled in appendix $\mathrm{B}$. It is noted that $\theta_{i}^{*, i}$ depends on $\theta_{j}, j \neq i$. We say that the SON mechanisms are stable in parallel mode if there exists $\theta^{*}$ which is asymptotically stable for (2). Typically, the SON mechanisms are designed and studied in a stand-alone manner, so that stand-alone stability is verified.

However, stand-alone stability does not imply parallel stability. First consider a case where $F_{i}(\theta)$ does not depend on $\theta_{j}$, for all $j \neq i$. Then (2) is a set of $I$ parallel independent ODEs, so that stand-alone stability implies parallel stability. On the other hand, if there exists $i \neq j$ such that $F_{i}(\theta)$ depends on $\theta_{j}$, then the situation is not so clear-cut. We say that SON $i$ and $j$ interact. Namely, interaction potentially introduces instablity.

In the remainder of this article we will be concerned with conditions for parallel stability, and designing coordination mechanisms to force stability whenever possible.

\section{B. Stability characterization}

Two particular cases of parallel mechanisms will be of interest. The first case is what we will call zero-finding algorithms. Each SON mechanism monitors the value of a performance indicator and tries to achieve a fixed target value for this performance indicator. Namely:

$$
F_{i}(\theta)=f_{i}(\theta)-\bar{f}_{i},
$$


where $f_{i}$ is the performance indicator monitored by SON $i$ and $\bar{f}_{i}$ - a target level for this performance indicator. The goal of the $i$-th SON mechanism is to find $\theta_{i}^{*}$ for fixed $\theta_{j}, j \neq i$ such that $f_{i}\left(\theta_{1}, \ldots, \theta_{i}^{*}, \ldots, \theta_{I}\right)=\bar{f}_{i}$. If $\theta_{i} \mapsto f_{i}\left(\theta_{1}, \ldots, \theta_{i}, \ldots, \theta_{I}\right)$ is strictly decreasing $1 \leq i \leq I$ then stand-alone stability is assured. Indeed, $V_{i}(\theta)=\left(f_{i}(\theta)-\bar{f}_{i}\right)^{2}$ would be a Lyapunov function for (3).

Another case of interest is maximization algorithms. Each SON mechanism tries to maximize a given performance indicator. There exists a continuously differentiable function $g_{i}$ such that:

$$
F_{i}(\theta)=\nabla_{\theta_{i}} g_{i}(\theta) .
$$

In stand-alone mode, SON $i$ indeed converges to a local maximum of $\theta_{i} \rightarrow g_{i}(\theta)$. If we restrict $\theta$ to a closed, convex and bounded set and if $\theta_{i} \mapsto g_{i}\left(\theta_{1}, \ldots, \theta_{i}, \ldots, \theta_{I}\right)$ is concave $1 \leq i \leq I$, we fall within the framework of concave games considered in [14]. Note that zero-finding algorithms can be rewritten as maximization algorithms by choosing $g_{i}(\theta)=-\left(f_{i}(\theta)-\bar{f}_{i}\right)^{2}$.

An important result of [14] for parallel stability is given in the following theorem. Denote by $w \in \mathbb{R}_{+}^{I}$ a vector of real positive weights.

Theorem 1. If $\sum_{i=1}^{I} w_{i} g_{i}(\theta)$ is diagonally strictly concave for $\theta$ in a convex bounded set $S \subset \mathbb{R}^{I}$, then the system of ODEs $\dot{\theta}=w^{T} \cdot F(\theta)$ admits a unique equilibrium point that is asymptotically stable in $S$.

If we denote by $J_{F, w}$ the Jacobian of $w \cdot F(\theta)=$ $\left[w_{1} F_{1}(\theta), \ldots, w_{I} F_{I}(\theta)\right]$, a sufficient condition for diagonal strict concavity is that $J_{F, w}+J_{F, w}^{T}$ is negative definite.

Note that 2) is a special case of the ODEs considered in Theorem 1 with $w_{i}=1, i=1, \ldots, I$. Without diagonal strict concavity there is no guarantee that parallel stability occurs, and coordination is needed.

\section{Linear case}

In the remainder of this paper, we study the case where $F$ is affine:

$$
F(\theta)=A \theta+b,
$$

with $b$ a vector of size $I$ and $A$ a matrix of size $I \times I$. We assume that $A$ is invertible and we define $\theta^{*}=-A^{-1} b$. The SON mechanisms running in parallel are described by the linear ODE:

$$
\dot{\theta}=A \theta+b=A\left(\theta-\theta^{*}\right) .
$$

It is noted that in the linear case, we always fall within the scope of zero-finding algorithms described previously, by defining:

$$
\begin{gathered}
f_{i}(\theta)=\sum_{\substack{1 \leq j \leq I \\
\dot{\theta}_{i}=f_{i}(\theta)-\bar{f}_{i} .}} A_{i, j} \theta_{j}, \bar{f}_{i}=-b_{i},
\end{gathered}
$$

In particular, stand-alone stability occurs if and only if (iff) $A_{i, i}<0,1 \leq i \leq I$, i.e all the diagonal terms of $A$ are strictly negative. Basic results on linear ODEs are recalled in appendix C Namely, parallel stability holds iff all the eigenvalues of $A$ have a strictly negative real part. We then say that $A$ is a Hurwitz matrix.

For practical systems, performance indicators $F(\theta)$ need not be linear functions of $\theta$. However, as long as they are smooth, they can be approximated by linear functions using a Taylor expansion in the neighborhood of a stationary point assuming a Taylor expansion exists. Consider a point $\theta^{*}$ with $F\left(\theta^{*}\right)=0$. If the values of $\theta$ are restricted to a small neighborhood of $\theta^{*}$ :

$$
F(\theta) \approx J F\left(\theta^{*}\right)\left(\theta-\theta^{*}\right)
$$

with $J F\left(\theta^{*}\right)$ the Jacobian of $F$ evaluated at $\theta^{*}$. The HartmanGrossman theorem ([15]) states that on a neighborhood of $\theta^{*}$, stability of the system with linear approximation implies stability of the original, non-linear system. Hence implementing the proposed coordination mechanism where $A$ is replaced by $J F$ ensures stability if we constrain $\theta$ to a small neighborhood of $\theta^{*}$.

The parameters $A$ and $b$ might be unknown, and we can only observe noisy values of $F(\theta)$ for different values of $\theta$. The crudest approach is to estimate $A$ and $b$ through finite differences:

$$
\begin{aligned}
a_{i, j} & \approx \frac{f_{j}\left(\theta+e_{i} \delta \theta_{i}\right)-f_{j}\left(\theta-e_{i} \delta \theta_{i}\right)}{2 \delta \theta_{i}}, \\
b_{i} & \approx f_{i}(0) .
\end{aligned}
$$

with $e_{i}$ the $i$-th unit vector. The results are averaged over several successive measurements and additive noise is omitted for notation clarity. In general, the measurements of $F$ are obtained by calculating the time average of some output of the network during a relatively long time, so that a form of the central limit theorem applies and the additive noise is Gaussian. In this case, a better method is to employ least-squares regression. Least-squares regression is a well studied topic with very efficient numerical methods ([16]) even for large data sets so that estimation of $A$ and $b$ is not computationally difficult.

Finally, since practical systems do not remain stationary for an infinite amount of time, a database with values of $A$ and $b$ for each set of operating conditions must be maintained. In the context of wireless networks, the relationship between parameters and performance indicators changes when the traffic intensity changes because of daily traffic patterns. For instance, during the night traffic is very low, and traffic peaks are observed at the end of the day. A database with estimated values of $A$ and $b$ at (for instance) each hour of the day should be constructed.

\section{COORDINATION}

\section{A. Coordination mechanism}

If $A$ has at least one eigenvalue with positive or null real part, convergence to $\theta^{*}$ does not occur, and a coordination mechanism is needed. We consider a linear coordination mechanism, where $A$ is replaced by $C A$ with $C$ a $I \times I$ real matrix. The ODE for the coordinated system is:

$$
\dot{\theta}=C A\left(\theta-\theta^{*}\right) \text {. }
$$


The coordination mechanism can be interpreted as transforming the performance indicator monitored by SON $i$ from $f_{i}$ to a linear combination of the performance indicators monitored by all the SON mechanisms. As explained in appendix C, stability is achieved if there exists a symmetric matrix $X$ such that:

$$
(C A)^{T} X+X C A \prec 0, \quad X \succ 0,
$$

where $X \succ 0$ denotes that $X$ is positive definite. In particular,

$$
V(\theta)=\left(\theta-\theta^{*}\right)^{T} X\left(\theta-\theta^{*}\right)
$$

acts as a Lyapunov function.

\section{B. Distributed implementation}

The choice for the coordination matrix $C$ is not unique. For instance $C=-A^{-1}$ ensures stability. For the coordination mechanism to be scalable with respect to the number of SONs, $C$ should be chosen to allow distributed implementation. We say that SON $j$ is a neighbor of SON $i$ if $\frac{\partial f_{j}}{\partial \theta_{i}} \neq 0$. We define $\mathcal{I}_{i}$ the set of neighbors of $i$. The coordination mechanism is distributed if each SON needs only to exchange information with its neighbors.

We give an example of a coordination mechanism which can always be distributed. The mechanism is based on a separable Lyapunov function. Define the weighted square error:

$$
V(\theta)=\sum_{i=1}^{I} w_{i}\left(f_{i}(\theta)-\bar{f}_{i}\right)^{2}=\left(\theta-\theta^{*}\right)^{T} A^{T} W A\left(\theta-\theta^{*}\right),
$$

with $W=\operatorname{diag}(w)$ i.e the diagonal matrix with diagonal elements $\left\{w_{i}\right\}_{1 \leq i<I}$. Coordination is achieved by following the gradient of $-V$ so that $V$ is a Lyapunov function:

$$
\dot{\theta}=-\nabla_{\theta} V(\theta)=-A^{T} W A\left(\theta-\theta^{*}\right) .
$$

Namely, we choose $C=-A^{T} W$. We can verify that the mechanism is distributed:

$$
\dot{\theta}_{i}=\sum_{j=1}^{I} 2 w_{i} \frac{\partial f_{j}}{\partial \theta_{i}}\left(f_{j}(\theta)-\bar{f}_{j}\right)=\sum_{j \in \mathcal{I}_{i}} 2 w_{j} \frac{\partial f_{j}}{\partial \theta_{i}}\left(f_{j}(\theta)-\bar{f}_{j}\right)
$$

Indeed, the update of $\theta_{i}$ only requires knowledge of $\frac{\partial f_{j}}{\partial \theta_{i}}$ and $f_{j}(\theta)-\bar{f}_{j}$, for $j \in \mathcal{I}_{i}$, and this information is available from the neighbors of $i$.

It is also noted that such a mechanism can be implemented in an asynchronous manner, i.e the components of $\theta$ are updated in a round-robin fashion, or at random instants, and the average frequency of update is the same for all components. The reader can refer to [17][chapters 6-8] for the round-robin updates and [18][chapter 12] for the random updates. Asynchronous implementation is important in practice because if the SONs are not co-located, maintaining clock synchronization among the SONs would generate a considerable amount of overhead.

\section{Stochastic Control Stabilization}

In practical systems, ODEs are replaced by stochastic approximation algorithms. Indeed, the variables are updated at discrete times proportionally to functions values which are noisy.

The noise in the function values is due to the fact that time is slotted and functions are estimated by averaging certain counters during a time slot. For example, the load of a BS in a mobile network is often estimated by evaluating the proportion of time during which the scheduler is busy, and the file transfer time is estimated by averaging the file transfer times of all flows occurring in a certain period of time. The noise is also due to intrinsic stochastic nature of real systems, for example in wireless networks the propagation environment is inherently non-deterministic (because of fading, mobility, etc.) so the Signal to Interference plus Noise Ratio (SINR) will be noisy.

When the noise in the measurements of the function values is of Martingale difference type, the mean behavior of those Stochastic Approximation (SA) algorithms matches with the system of ODEs. Note that we consider Martingale difference type of noise but the SA results hold for much more general noise processes (stationary, ergodic). In [10] for example, SA results are used without the Martingale difference property.

The initial system of control loops is in reality a system of $\mathrm{SA}$ algorithms, with one of them written as

$$
\theta_{i}[k+1]=\left[\theta_{i}[k]+\epsilon_{k}\left(f_{i}(\theta[k])+N_{k}^{i}\right)\right]_{S_{i}}^{+}
$$

where $[.]_{S_{i}}^{+}$is the projection on the interval $S_{i}=\left[a_{i}, b_{i}\right] ; a_{i}<$ $b_{i} \in \mathbb{R}, \theta[k]=\left(\theta_{1}[k], \ldots, \theta_{I}[k]\right)$ is the vector of parameters after the $(k-1)$ th update, $\epsilon_{k}$ the step of the $k$ th update and $N_{k}^{i}$ represents the noise in the measurement.

The projection in (18) aims at ensuring that the iterates are bounded. This is also a condition for convergence of the SA algorithm towards the invariant sets of the equivalent ODE.

Most SON algorithms are or can be reduced to the form of (18). For example in [3], a load balancing SON is presented in this very same form. In [10] relays are self-optimized using also a SA algorithm. In [19], SA algorithms are used for self-optimizing interference management for femtocells. A handover optimization SON which can be rewritten as an SA algorithm is also presented in [20].

We suppose that $N_{k}$ is a martingale difference sequence to meet the conditions for stand alone convergence (see [18], [21]). Namely the SA algorithms have the same behavior as their equivalent ODE. Now we want to check if the conditions for the SA equivalence with the limiting ODE are still verified after the coordination mechanism is applied. The coordinated $\mathrm{SA}$ for the $i$-th mechanism is

$$
\theta_{i}[k+1]=\left[\theta_{i}[k]+\epsilon_{k}\left(\sum_{j=1}^{I} C_{i, j}\left(f_{j}(\theta[k])+N_{k}^{j}\right)\right)\right]_{S_{i}}^{+}
$$

The projection ensures that the iterates are bounded. The question now is to show that $\sum_{j=1}^{I} C_{i, j} N_{k}^{j}$ is a Martingale 
difference sequence in order to meet the convergence conditions. Denoting $\mathcal{F}_{k}=\left\{\sum_{j=1}^{I} C_{i, j} N_{l}^{j}, l<k\right\}$, we have

$$
E\left[\sum_{j=1}^{I} C_{i, j} N_{k}^{j} \mid \mathcal{F}_{k}\right]=\sum_{j=1}^{I} C_{i, j} E\left[N_{k}^{j} \mid \mathcal{F}_{k}\right]=0
$$

since $E\left[N_{k}^{j} \mid N_{l}^{j}, l<k\right]=0, j=1 \ldots I$. So this condition is satisfied ensuring the validity of the coordination method in a stochastic environment.

\section{Fully distributed COORDinAtion}

In this section we study fully distributed coordination, where the coordination matrix $C$ is diagonal. As said previously, if $C_{i, j} \neq 0, i \neq j$ then SON $i$ and $j$ need to exchange information. In fully distributed coordination, no information is exchanged. We prove two results. For $I=2$ fully distributed coordination can always be achieved. For $I=3$ it is also possible if $A^{-1}$ has at least one non-zero diagonal element and impossible otherwise. These results are attractive from a practical point of view: if there are 3 or less SONs to coordinate, it suffices to modify their feedback coefficient, without any exchange of information or interface between them. We say that the system can be coordinated in a fully distributed way iff there exists $c \in \mathbb{R}^{I}$ such that $\operatorname{diag}(c) A$ is a Hurwitz matrix.

The following lemma will be useful. It is a consequence of the Routh-Hurwitz theorem ([22]).

Lemma 1. Let $M$ a $I \times I$ invertible real matrix. For $I=2$, $M$ is a Hurwitz matrix iff

$$
\operatorname{det}(M)>0, \operatorname{tr}(M)<0
$$

where $\mathrm{tr}$ denotes the trace of a matrix.

For $I=3, M$ is a Hurwitz matrix iff

$$
\operatorname{det}(M)<0, \operatorname{tr}(M)<0, \operatorname{tr}(M) \operatorname{tr}\left(M^{-1}\right)>1 .
$$

For $I=2$ mechanisms, the system can always be coordinated in a fully distributed way as shown by Theorem 2

Theorem 2. For $I=2$, the system can always be coordinated in a fully distributed way. $\operatorname{diag}(c) A$ is a Hurwitz matrix iff:

$$
c \in \mathcal{C}=\left\{c: c_{1} A_{1,1}+c_{2} A_{2,2}<0, c_{1} c_{2} \operatorname{det}(A)>0\right\},
$$

and $\mathcal{C}$ is not empty since:

$$
\left(1, \text { sign } \operatorname{det}(A) \frac{\left|A_{1,1}\right|}{2\left|A_{2,2}\right|}\right) \in \mathcal{C} .
$$

Proof: $\operatorname{tr}(\operatorname{diag}(c) A)=c_{1} A_{1,1}+c_{2} A_{2,2} \quad$ and $\operatorname{det}(\operatorname{diag}(c) A)=c_{1} c_{2} \operatorname{det}(A)$. Using Lemma 11 proves the first part of the result. $\mathcal{C}$ is not empty, since one of its elements is given by inspection of the proposed value.

For $I=3$ mechanisms, the system can also be coordinated in a fully distributed way providing that the inverse of $A$ has one non-zero diagonal element as shown by Theorem 3

Theorem 3. For $I=3$, the system can be coordinated in a fully distributed way if $B=A^{-1}$ has at least one non-zero diagonal element. Assume that $B_{2,2} \neq 0$ without loss of generality. Consider $\epsilon>0$, and define $C(\epsilon)=\operatorname{diag}\left(1, \epsilon c_{2}, \epsilon c_{3}\right)$ with:

$$
\frac{B_{2,2}}{c_{2}}+\frac{B_{3,3}}{c_{3}}<0, c_{2} c_{3} \operatorname{det}(A)<0 .
$$

$A$ possible choice for $\left(c_{2}, c_{3}\right)$ is $c_{2}=-B_{2,2}$ and $c_{3}=-2 \operatorname{sign}\left(\operatorname{det}(A) c_{2}\right)\left|B_{3,3}\right|$ if $B_{3,3} \neq 0$ and $c_{3}=$ $-\operatorname{sign}\left(\operatorname{det}(A) c_{2}\right)$ otherwise.

Then there exists $\epsilon_{0}$ such that $C(\epsilon) A$ is a Hurwitz matrix for $0<\epsilon<\epsilon_{0}$.

If $B$ has a null diagonal, the system cannot be coordinated.

Proof: We have that

$$
\begin{aligned}
\operatorname{tr}(C(\epsilon) A) & =A_{1,1}+O(\epsilon), \\
\operatorname{tr}(C(\epsilon) A) \operatorname{tr}\left((C(\epsilon) A)^{-1}\right) & =\frac{A_{1,1}}{\epsilon}\left(\frac{B_{2,2}}{c_{2}}+\frac{B_{3,3}}{c_{3}}\right)+O(1) .
\end{aligned}
$$

Recall that $A_{1,1}<0$. So there exists $\epsilon_{0}$ such that $\operatorname{tr}(C(\epsilon) A)<$ 0 and $\operatorname{tr}(C(\epsilon) A) \operatorname{tr}\left((C(\epsilon) A)^{-1}\right)>1$, if $\epsilon>\epsilon_{0}$. Using Lemma 1. $C(\epsilon) A$ is a Hurwitz matrix for $0<\epsilon<\epsilon_{0}$. The existence of a couple $\left(c_{2}, c_{3}\right)$ is given by inspection of the proposed value. If $B$ has a null diagonal, then $\operatorname{tr}\left((\operatorname{diag}(c) A)^{-1}\right)=0$ for all $c$, so that the conditions of Lemma 1 can never be met.

For $I>3$, the problem becomes more involved. Sufficient conditions for the existence of a diagonal matrix can be found in the literature. In particular Fisher and Fuller (1958) [23] have proven that if there exists a permutation matrix $\mathrm{P}$ such that all leading principal sub-matrices of $\hat{A}=P A P^{-1}$ are of full rank, then A can be stabilized by scaling.

A more restrictive version of this condition which gives a simple way to construct the coordination matrix is given in the following theorem.

Theorem 4. If all leading principal sub-matrices of $A$ are of full rank, then there exists a diagonal matrix $C=$ $\operatorname{diag}\left(c_{1}, c_{2}, . ., c_{N}\right) \in \mathbb{R}^{I \times I}$ that stabilizes $A$ (i.e. $C A$ is Hurwitz).

Proof: Indeed, it then suffices to choose $c_{1}, c_{2}, \ldots, c_{I}$ sequentially such that $(-1)^{i} c_{1} \ldots c_{i} \operatorname{det}\left([A]_{i, i}\right)>0$ for $i=$ $1, \ldots, I$ where $[A]_{i, i}$ is the submatrix of $A$ comprised of lines 1 trough $i$ and columns 1 through $i$. This means that $\forall k=1 . . I$, $(-1)^{k} \operatorname{det}\left([C A]_{k, k}\right)>0$ which implies by a known result $[24$ Section 16.7] on negative definite matrices that all eigenvalues of $C A+(C A)^{T}$ are strictly negative.

Later works have extended the Fisher and Fuller condition to more general cases [25].

\section{COORDINATION AS A CONVEX OPTIMIZATION PROBLEM}

This section aims to formalize the problem of finding a coordination matrix $C$ such that $(12)$ is stable when (6) is not. We begin by recalling a sufficient condition for stability mentioned in Section II-B, declining it for the linear case.

Theorem 5. Suppose there exists a $I \times I$ matrix $C$ verifying

$$
(C A)^{T}+C A \prec 0,
$$


then $A$ and $C$ are invertible, $\theta^{*}$ is the only equilibrium point of (12) and it is globally asymptotically stable.

Proof: If $C A+(C A)^{T} \prec 0$, then CA is invertible, and so the equation $C F(\theta)=C A\left(\theta-\theta^{*}\right)=0$ has a unique solution which is $\theta^{*}$.

The global asymptotic stability is obtained from Theorem 1. since condition 25 implies diagonal strict concavity.

Note that $\theta^{*}$ is also an equilibrium point of (6). In addition to the constraint 25] we need to consider an additional constraint which is related to the capability of the different SON entities to exchange information. For example, if two SONs $i, j$ are located at different BSs in a LTE network without a X2 interface between them, then the element $C_{i, j}$ in matrix $C$ must be equal to 0 . On the other hand, if $C_{i, j} \neq 0$, then updating the parameter $\theta_{i}$ requires the value of $f_{j}(\theta)$, so we have to be sure that this information can be made available. Typically in a network for example, this relates to interfaces that exist between BSs, so the system constraints will be mapped from the network architecture. To add this constraint, we define a set of system-feasible matrices in $\mathbb{R}^{N \times N}$, called $\mathcal{C}$ reflecting our system constraints.

Denote the two constraints mentioned above stability and connectivity constraints. These two constraints may be verified by a large number of matrices, and the one with the best convergence properties is sought. From convex optimization theory, we know that iterative algorithms converge faster when their condition number gets lower [26]. Indeed, the solution of the system of ODEs $\dot{x}=C A x$ is in fact $x(t)=e^{t C A} x_{0}$. The exponential of a matrix is defined using the power series. The solution is then rewritten as

$$
x(t)=\left(\sum_{k=0}^{\infty} \frac{t^{k}}{k !}(C A)^{k}\right) x_{0}
$$

Now if we choose $x_{0}$ as an eigenvector of $C A$ with the eigenvalue $\lambda_{0}$, we can see that $x(t)=\left(\sum_{k=0}^{\infty} \frac{t^{k}}{k !} \lambda_{0}^{k}\right) x_{0}=e^{\lambda_{0} t} x_{0}$. Now this is true for all the eigenvectors of the matrix $C A$ so that for a random starting point $x_{0}$, a lower condition number will ensure a better convergence as the speed of convergence will be homogeneous across the eigenspaces.

So without constraints, the best coordination matrix would be $-A^{-1}$, leading to a diagonal matrix $C A=-I_{N}$ which gives us a system with the lowest condition number i.e. 1 . When taking the constraints into account, we formulate the convex optimization problem as minimizing the distance, defined in terms of the Frobenius norm, between the coordination matrix $C$ and $-A^{-1}$ :

$$
\begin{aligned}
& \text { minimize }\left\|C+A^{-1}\right\|_{F} \\
& \text { s.t. }(C A)^{T}+C A \prec 0 ; C \in \mathcal{C}
\end{aligned}
$$

where $\|.\|_{F}$ is the Frobenius norm defined for an $\mathbb{R}^{m \times n}$ matrix $M$ as

$$
\|M\|_{F}=\sqrt{\sum_{i=1}^{m} \sum_{j=1}^{n}\left|M_{i, j}\right|^{2}}=\sqrt{\operatorname{Tr}\left(M^{T} M\right)}=\sqrt{\sum_{i=1}^{\min (m, n)} \sigma_{i}^{2}}
$$

with the $\sigma_{i}$ being the singular values of $M$. It is noted that the Frobenius norm is often used in the literature for finding a preconditioner that improves the convergence behavior of iterative inversion algorithms [27].

The stability constraints are expressed in the form of LMIs. LMIs are a common tool used in control theory for assessing stability. Solving convex optimization problems with LMI constraints is a tractable problem and fast solvers are available [28].

From the implementation point of view, the coordination process can be performed in two steps as follows. In the first step, a centralized coordination server gathers and processes data to derive the matrix $A$, performs the optimization problem (26) to obtain the coordination matrix $C$, and sends each line of the matrix $C$ to the corresponding SON entity. This step is performed off-line. The second step is the on-line control process where each SON performs the coordinated control, while satisfying the connectivity constraints, by using the appropriate line of matrix $C$.

\section{APPLICATION TO WIRELESS NETWORKS}

In this section we illustrate instability and coordination in the context of wireless networks using two examples. We first show that instability occurs even in simple models with as few as two SONs in parallel. Then we apply the coordination to a use case involving 3 SONs deployed in several BSs in a LTE network.

\section{A. Admission control and resource allocation}

1) Model: We consider a BS in downlink, serving elastic traffic. Users enter the network according to a Poisson process with arrival rate $\lambda$, to download a file of exponential size $\sigma$, with $\mathbb{E}[\sigma]<+\infty$. The BS has $x_{\max }$ parallel resources available, and we write $x \in\left[0, x_{\max }\right]$ the amount of resources used. We ignore the granularity of resources, either assuming that there are a large number of resources or using time sharing, using each resource a proportion $\frac{x}{x_{\max }}$ of the time. Depending on the access technology, resources can be: codes in Code Division Multiple Access (CDMA), time slots in Time Division Multiple Access (TDMA), time-frequency blocks in Orthogonal Frequency-Division Multiple Access (OFDMA) etc. When a user is alone in the system, his data rate is $R x$. Users are served in a processor sharing manner (for instance through Round Robin scheduling): if there are $n$ active users, each user has a throughput of $\frac{x R}{n}$. Admission control applies. We define $\beta \geq 0$ a blocking threshold and the probability of accepting a new user when $n$ users are already present in the system is $\phi(n-\beta)$ with $\phi: \mathbb{R} \rightarrow[0,1]$ a smooth, strictly decreasing function and $\phi(n) \underset{n \rightarrow+\infty}{\rightarrow} 0$. We choose $\phi$ as a logistic function for numerical calculations:

$$
\phi(n)=\frac{1}{1+e^{n}}
$$

Define $n(t)$ the number of active users in the system at time $t$, then $n(t)$ is a continuous time Markov chain. $n(t)$ is ergodic 
because the probability of accepting a new user goes to 0 as $n \rightarrow \infty$. We define the load:

$$
\rho(x)=\frac{\lambda \mathbb{E}[\sigma]}{x R} .
$$

We write $\pi$ the stationary distribution of $n(t) . n(t)$ is reversible, and $\pi$ can be derived from the detailed balance conditions:

$$
\pi(n, x, \beta)=\frac{\rho(x)^{n} \prod_{k=0}^{n-1} \phi(k-\beta)}{\sum_{n \geq 0} \rho(x)^{n} \prod_{k=0}^{n-1} \phi(k-\beta)} .
$$

Using Little's law, the mean file transfer time is given by the average number of active users divided by the arrival rate:

$$
T(x, \beta)=\frac{1}{\lambda} \sum_{n \geq 0} n \pi(n, x, \beta) .
$$

Let $R_{\min }$ a minimal data rate required to ensure good Quality of Service (QoS). We say that there is an outage in a state of the system if users have a throughput lower than $R_{\min }$. When there are $n$ active users in the system, outage occurs iff:

$$
n>\frac{x R}{R_{\min }} \text {. }
$$

The outage probability is then:

$$
O(x, \beta)=\sum_{n \geq 0} \pi(n, x, \beta) \mathbf{1}_{(0,+\infty)}\left(n-\frac{x R}{R_{\text {min }}}\right) .
$$

In this model, $x \rightarrow O(x, \beta)$ is not smooth, which is why we introduce the smoothed outage $\tilde{O}$ :

$$
\tilde{O}(x, \beta)=\sum_{n \geq 0} \pi(n, x, \beta) \psi\left(n-\frac{x R}{R_{\text {min }}}\right) .
$$

with $\psi$ a smooth function approximating $\mathbf{1}_{(0,+\infty)}$. We also choose $\psi$ as a logistic function for numerical calculations.

This queuing system is controlled by two mechanisms, and that control occurs on a time scale much slower than the arrivals and departures of the users, so that the mean file transfer time and outage probability are relevant performance metrics, and can be estimated from (noisy) measurements. The mechanisms are:

- Resource allocation: a mechanism adjusts the amount of used resources to reach a target outage rate. Such mechanisms have been considered in green networking when a BS can switch off part of its resources in order to save energy.

Another application is interference coordination: using more resources will create inter-cell interference in neighboring BSs and degrade their QoS. Hence BSs should use as little resources as possible, as long as their target QoS is met.

- Admission control: another mechanism adjusts the admission control threshold to reach a target file transfer time. In particular, it is noted that without admission control, the mean file transfer time becomes infinite in overload.
It is noted that $x \rightarrow \tilde{O}(x, \beta)$ is strictly decreasing and $\beta \rightarrow T(x, \beta)$ is strictly increasing. Using the notations of Section II we have $I=2$ control loops, $\theta_{1} \equiv x, \theta_{2} \equiv \beta$, $f_{1} \equiv \tilde{O}, f_{2} \equiv-T$. Consider $\theta^{*}=\left(x^{*}, \beta^{*}\right)$ an operating point The stability in the neighborhood of $\left(x^{*}, \beta^{*}\right)$ can be calculated. The system will fail to converge to the desired operating point as long as the Jacobian matrix has a negative determinant, so that there are two eigenvalues of opposite sign:

$$
-\frac{\partial \tilde{O}}{\partial x} \frac{\partial T}{\partial \beta}\left(x^{*}, \beta^{*}\right)+\frac{\partial \tilde{O}}{\partial \beta} \frac{\partial T}{\partial x}\left(x^{*}, \beta^{*}\right)<0
$$

2) Results: We now evaluate the stability region of the system numerically by checking condition (35) for various operating points. We choose the following parameter values: $\lambda=0.5$ users $/ \mathrm{s}, \mathbb{E}[\sigma]=10 \mathrm{Mbits}, R=15 \mathrm{Mbits} / \mathrm{s}$, $R_{\min }=2 \mathrm{Mbits} / \mathrm{s}, x_{\max }=1$. Figure 1 presents the results. In the white region the system is stable, and in the gray region it is unstable. Even in such a simple setting with 1 BS and 2 SON mechanisms, instability occurs for a large set of operating points.

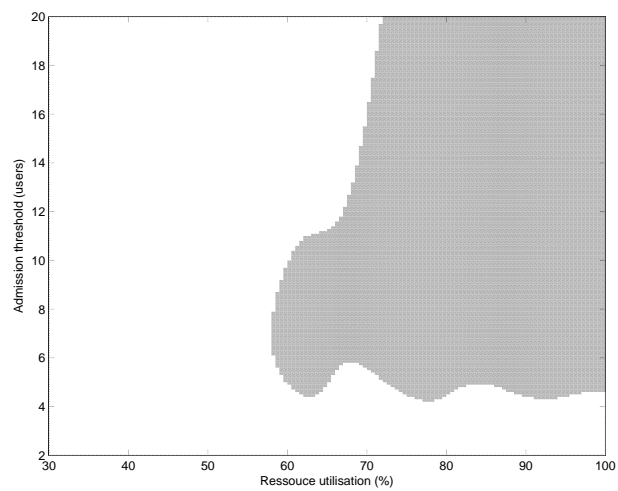

Fig. 1. Stability region of the system

\section{B. SON Coordination use case}

1) System Model: Consider three SON mechanisms deployed in BSs of a LTE network: blocking rate minimization, outage probability minimization and load balancing SON as presented in [3]. We focus on downlink ftp type traffic model in which each user enters the network, downloads a file from its serving cell and then leaves the network.

a) Load balancing: The SON adjusts the BS's pilot powers in order to balance the loads between neighboring cells. The corresponding ODE is given by

$$
\dot{P}_{s}=P_{s}\left(\rho_{1}(\mathbf{P})-\rho_{s}(\mathbf{P})\right), \forall s=1 \ldots I
$$

where $\mathbf{P} \in \mathbb{R}^{I}$ is the vector of BSs' pilot powers, and $\rho$ - their corresponding loads. This SON converges to a set on which all loads are equal as shown in [3, Theorem 4]. We use an equivalent formulation in order to update the pilots directly in $\mathrm{dB}$ :

$$
\dot{P}_{\mathrm{dB}_{s}}=\rho_{1}(\mathbf{P})-\rho_{s}(\mathbf{P}) \forall s=1 \ldots I .
$$

where $P_{\mathrm{dB} s}$ is the pilot power of BS $s$ in decibels. 
b) Blocking rate minimization: This SON adjusts the admission threshold in order to reach a given blocking rate target $\bar{B}>0$. Denote by $x_{s} \in \mathbb{R}^{+}$a real value whose floor (i.e. the smaller integer part) equals the effective admission threshold of BS $s$, i.e. a new user finding the cell with $n$ users is blocked with probability $P(n)$, where $P(n) \rightarrow 1$ when $n \rightarrow x_{s}$ and $P(n) \rightarrow 0$ when $n \rightarrow 0$. The update equation for the blocking rate minimization $\mathrm{SON}$ is

$$
x_{s, t+1}=\left[x_{s, t}+\epsilon_{t}\left(B_{s}\left(x_{t}\right)-\bar{B}_{s}+N_{t}\right)\right]_{\left[0, x_{\max }\right]}^{+}
$$

where $x_{t}$ is the vector of the admission thresholds of all the BSs considered at time $\mathrm{t}, x_{\max }$ a sufficiently large value and $N_{t}$ a martingale difference noise introduced by measuring $B\left[x_{t}\right]$. The equivalent ODE is

$$
\dot{x}_{s}=B_{s}(x)-\bar{B}_{s} .
$$

$x_{s} \rightarrow B_{s}(x)$ is a decreasing function of $x_{s}$ and $\lim _{x_{s} \rightarrow \infty} B_{s}(x)=0$. So for any blocking rate target $0<$ $\bar{B}_{s}<1$, we have $B_{s}(0) \geq \bar{B}_{s}$ and there exists a finite $x_{0} \in \mathbb{N}$ such that $\forall x \geq x_{0} ; B_{s}(x) \leq \bar{B}$ and $\forall x \leq x_{0} ; B(x) \geq \bar{B}$. By projecting the right hand side of (38) on any interval containing $\left[0, x_{0}\right]$, we ensure that $\sup _{t}\left\|x_{t}\right\|<\infty$.

Now considering the function $V(x)=\max \left(0,\left|x-x_{0}\right|-\delta\right)$ for $\delta$ sufficiently small, we can see that $V($.$) is a Lyapunov$ function for (39). Indeed, we have

- $\forall x \in[0,+\infty), V(x) \geq 0$.

- $H=\{x \in[0,+\infty), V(x)=0\} \neq \emptyset$ because it contains $x_{0}$.

$$
\begin{aligned}
\text { - } \dot{V}(x) & =\frac{\partial V}{\partial x} \dot{x} \\
& = \begin{cases}-(B(x)-\bar{B}) & \text { if } x<x_{0}-\delta \\
B(x)-\bar{B} & \text { if } x>x_{0}+\delta \\
0 & \text { if } x \in\left[x_{0}-\delta, x_{0}+\delta\right]\end{cases} \\
& \leq 0 .
\end{aligned}
$$

- $V(x) \stackrel{\rightarrow}{\rightarrow}+\infty$ when $x \rightarrow+\infty$.

This implies that $H$ is globally asymptotically stable for 39 . (see Appendix B).

c) Outage Probability Minimization: The aim of this SON mechanism is to adjust the transmit data power in order to reach a target outage probability. The outage probability considered is expressed as

$$
K_{s}=\frac{1}{\left|Z_{s}\right|} \int_{Z_{s}} \mathbb{1}_{\left\{R_{s}(r) \geq R_{\min }\right\}}(r) d r
$$

where $Z_{s}$ is the area covered by BS $s, R_{\min }$ a minimum data rate and $R_{s}(r)$ the peak data rate obtained at position $r$ when served by BS $s$. The SA algorithm modeling the actual control loop is

$$
D_{s}[k+1]=D_{s}[k]-\epsilon_{k}\left(K_{s}(\mathbf{D}[k])-\bar{K}+N_{k}^{s}\right)
$$

\begin{tabular}{|c|c|}
\hline \multicolumn{2}{|c|}{ Network parameters } \\
\hline Number of stations & 19 (with wrap-around) \\
\hline Cell layout & hexagonal omni \\
\hline Intersite distance & $500 \mathrm{~m}$ \\
\hline Bandwidth & $20 \mathrm{MHz}$ \\
\hline \multicolumn{2}{|c|}{ Channel characteristics } \\
\hline Thermal noise & $-174 \mathrm{dBm} / \mathrm{Hz}$ \\
\hline Path loss $(\mathrm{d}$ in $\mathrm{km})$ & $128+36.4 \log _{10}(d) \mathrm{dB}$ \\
\hline \multicolumn{2}{|c|}{ Traffic characteristics } \\
\hline Arrival rate & 40 users/s \\
\hline Service type & FTP \\
\hline Average file size & 10 Mbits \\
\hline Hotspot additional arrival rate & 2 users/s \\
\hline Hotspot position & center of BS 1 cell \\
\hline Hotspot diameter & $330 \mathrm{~m}$ \\
\hline \multicolumn{2}{|c|}{ Simulation parameters } \\
\hline Spatial resolution & $20 \mathrm{~m} \times 20 \mathrm{~m}$ \\
\hline Time per iteration & $6 \mathrm{~s}$ \\
\hline Minimun SINR for coverage & $0 \mathrm{~dB}$ \\
\hline Target outage probability & $82 \%$ \\
\hline Target blocking rate & $2 \%$ \\
\hline
\end{tabular}

where $N_{k}^{s}$ is a martingale difference noise and $D_{s}$ is the transmit data power of BS $s$. The limiting ODE representing the mean behaviour of SA (41) is then

$$
\dot{D}_{s}=-\left(K_{s}(D)-\bar{K}\right) \text {. }
$$

TABLE I. NETWORK AND TRAFFIC CHARACTERISTICS

This ODE is stable if there exists an admissible data power $D_{s}^{*}$ such that $K_{s}\left(D_{s}^{*}\right)=\bar{D}_{s}$. Indeed, $\left(K_{s}(.)-\bar{K}\right)^{2}$ would then be a Lyapunov function for 42 since $\frac{\partial K_{s}}{\partial D_{s}}>0$. As a consequence, the SA (41) converges to invariant sets of (42), which means that the mechanism is standalone-stable.

2) Numerical Results: Consider a hexagonal network with 19 cells with omni-directional antennas as shown in Fig2 A wrap-around model is used to minimize truncation effects of the computational domain. It is achieved by surrounding the original network with 6 of its copies while performing the simulation within the original 19 cells.

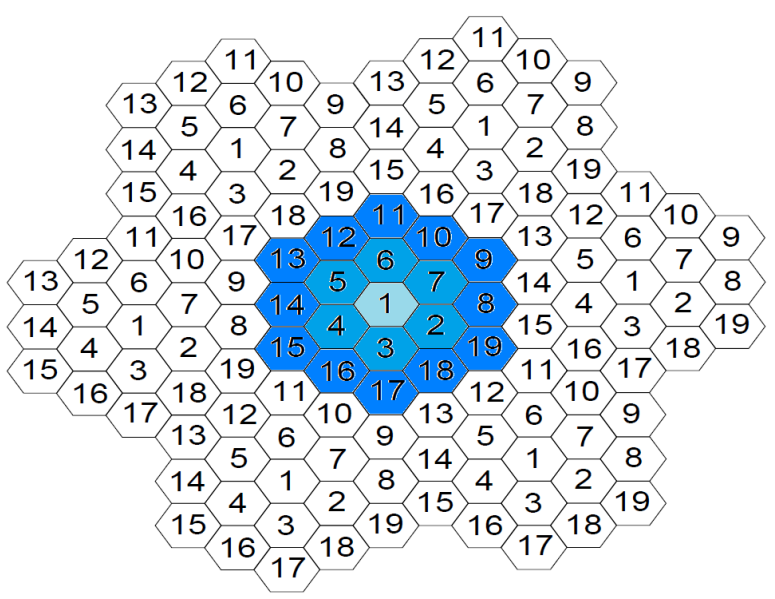

Fig. 2. 19 cells hexagonal network with wrap-around

Table I lists the parameters used in the simulations including the environment, the network, the numerical simulation parameters and the KPIs' targets used by the SON mechanisms. The 
users arrive in the network according to a Poisson process with a certain arrival rate given in Table I And a hotspot is placed at the center of the network with an additional arrival rate also given in Table 1 . The hotspot provides initially unbalanced loads in the network, which is of interest for the load balancing SON.

We activate the three SONs in each of the 7 BSs located at the center of the map and observe the stability of the SONs, with and without the coordination mechanism.

The first step consists in computing the stability matrix of the linearized control system. This step is performed by evaluating the closed-form formulas of the corresponding KPIs and then computing the partial derivatives using finite differences. By choosing an adequate step size, this method yields very accurate results. However, closed-form expressions of the KPIs do not always exist, in which case estimations of the KPIs would be used instead, based on measurements from each user that arrive in the network. The stability matrix obtained through linearization already reveals instability since not all of its eigenvalues are negative, and hence the coordination step is inevitable.

We plot the KPIs evolutions for the coordinated (in blue) and non-coordinated (in red) systems (Figures 3 to 5). The coordinated system clearly performs better. The loads are lower, the blocking rates too. The outage probabilities in the non-coordinated system diverge. The most loaded BS outage probability is near zero while that of the others reach the maximum. This is because the decrease in cell size of the most loaded BS is not followed by a decrease of its traffic power, causing more interference on its neighbors which have increased their cell size. The goals of each SON are more or less achieved in the coordinated system. And the loads are balanced very quickly (less than 10 minutes), this shows the usefulness of distributed SONs over centralized ones.

In Fig 4, we can see that the outage of BS 1 in the coordinated network is good but off the target we had set for the outage SON. This is a consequence of putting together many types of SONs which are not homogeneous. If needed, the SON entities can be harmonized by using some weights. We now investigate the impact of the weights on the stationary KPIs of the system.

Figures 6 and 7 compare the final values of the KPIs of the coordinated (in blue) and non-coordinated (in red) systems when they reach their permanent state for different weight vectors. For equal weight across all SONs, we can see in Fig 6 that the system is rather drifted towards balancing the loads as not all the BSs reach their outage target. Actually traffic power of BS 1 (the most loaded one) increases to absorb more traffic while its cell size is reduced leading to a smaller outage probability.

We then give more importance to outage target in the SONs by increasing 20 times its weight. In Figure 7 , we plot the numerical results obtained. The outages are closer to their goals but the system does not balance the loads anymore. This shows us that with the coordination, a compromise is made. This compromise can be changed by adjusting the weights of the SONs to reflect the policies or the priorities of the network operator.

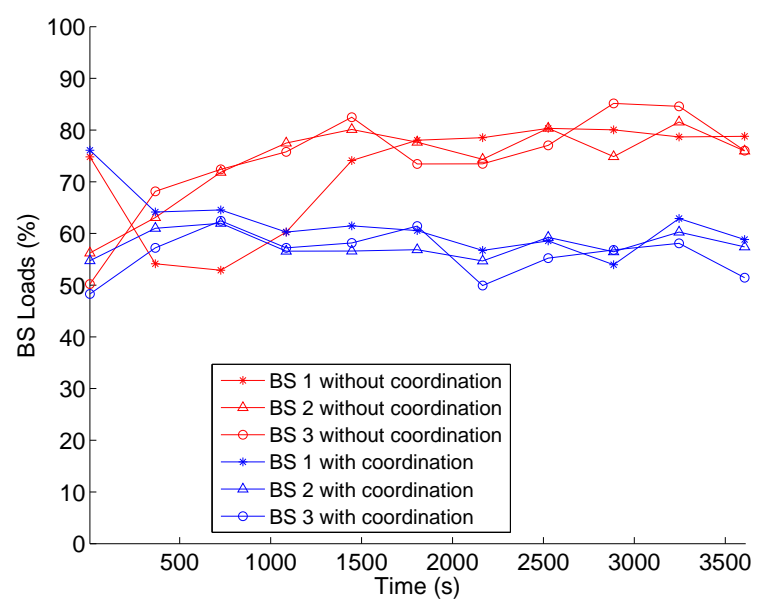

Fig. 3. Impact of Coordination on Loads

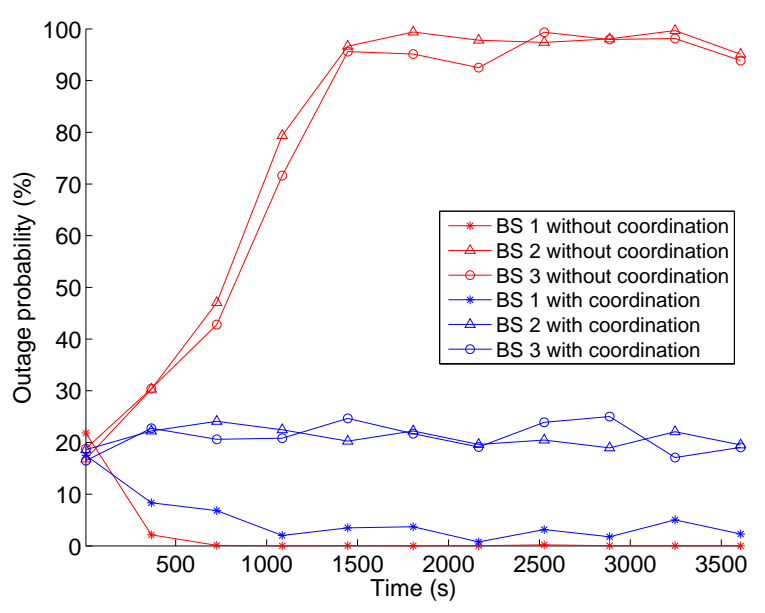

Fig. 4. Impact of Coordination on Coverage Probabilities

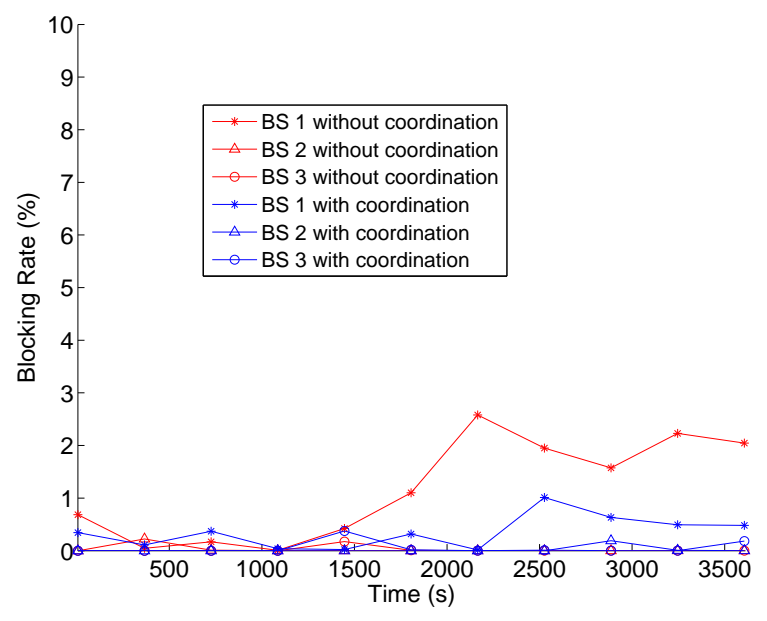

Fig. 5. Impact of Coordination on Blocking Rates 


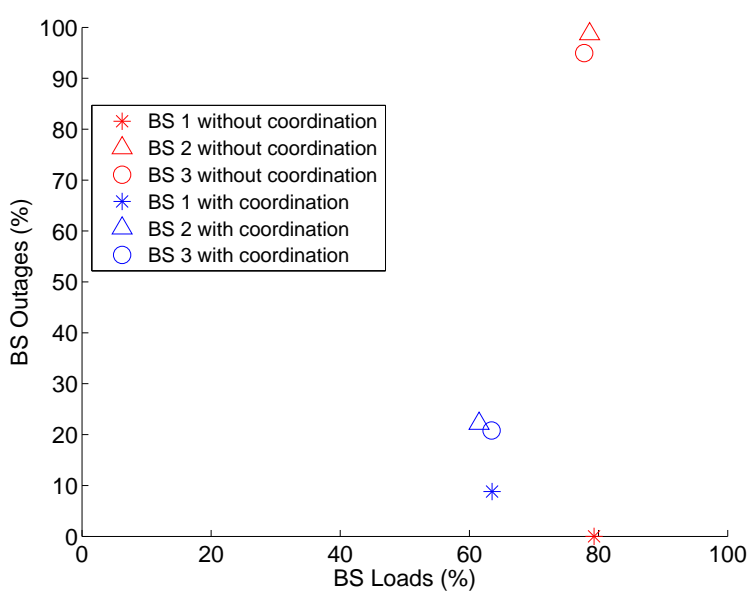

Fig. 6. Stationary KPIs with with all SONs equally weighted

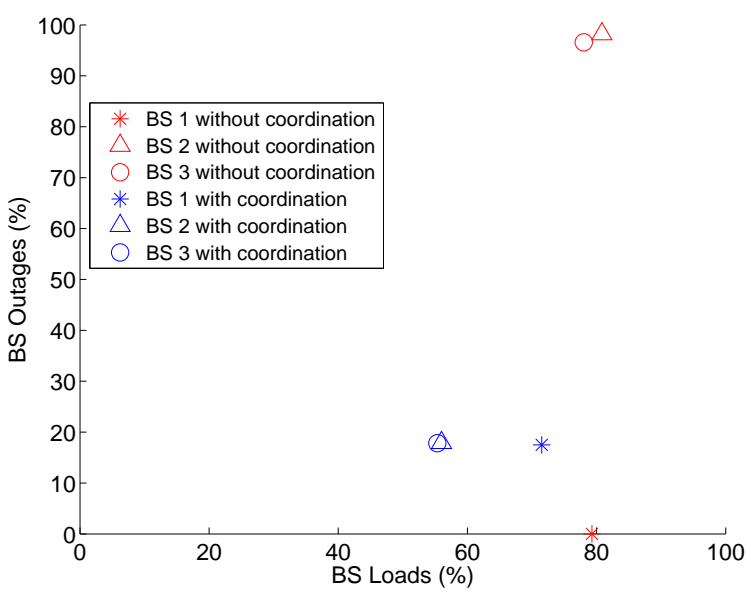

Fig. 7. Stationary KPIs with outage probability prioritized

\section{CONCLUding Remarks}

In this paper we have studied the problem of coordinating multiple SON entities operating in parallel. Using tools from control theory and Lyapunov stability, we have proposed a coordination mechanism to stabilize the system. The problem of finding a coordination matrix has been formulated as a convex optimization problem with LMIs constraints which ensures that the system of SONs remain distributed. The coordination can be implemented in a distributed fashion so it is scalable with respect to the number of SONs. We have also shown that the coordination solution remains valid in the presence of measurement noise, using stochastic approximation. Instability in the context of wireless networks have been illustrated with an example. We have shown that even for two control loops, instability can occur, and the influence of network geometry has been investigated. A practical use case of the coordination method has been presented in a LTE network implementing three distributed SON functions deployed in several base stations. The coordination reveals to be necessary in this use case since the system of SONs has shown to be unstable without coordination. The different SON entities achieve their respective goals when coordinated and the network is stabilized. This use case has also shown that in spite of the linear control assumption, the method remains effective when applied to SON functionalities that are not linear in general.

\section{APPENDIX A \\ PROOF OF LEMMA 1}

Proof: We denote by $P(\lambda)=\operatorname{det}\left(\lambda I_{I}-M\right)$ the characteristic polynomial of $M . M$ is a Hurwitz matrix iff $P$ has no roots with positive real part. For $I=2$,

$$
P(\lambda)=\lambda^{2}-\operatorname{tr}(M) \lambda+\operatorname{det}(M) .
$$

By the Routh-Hurwitz theorem, $P$ has no roots with positive real part iff:

$$
-\operatorname{tr}(M)>0,-\operatorname{tr}(M) \operatorname{det}(M)>0
$$

which proves the result. For $I=3$,

$$
P(\lambda)=\lambda^{3}-\operatorname{tr}(M) \lambda^{2}-\frac{\lambda}{2}\left(\operatorname{tr}\left(M^{2}\right)-\operatorname{tr}(M)^{2}\right)-\operatorname{det}(M) .
$$

By the Routh-Hurwitz theorem, $P$ has no roots with positive real part iff: $-\operatorname{tr}(M)>0,-\operatorname{det}(M)>0$ and:

$$
\frac{\operatorname{tr}(M)}{2}\left(\operatorname{tr}\left(M^{2}\right)-\operatorname{tr}(M)^{2}\right)+\operatorname{det}(M)>0 .
$$

For $I=3$, we have that:

$$
\operatorname{tr}\left(M^{2}\right)-\operatorname{tr}(M)^{2}=-2 \operatorname{det}(M) \operatorname{tr}\left(M^{-1}\right),
$$

so that the last condition reduces to

$$
\operatorname{tr}(M) \operatorname{tr}\left(M^{-1}\right)-1>0
$$

proving the result.

\section{APPENDIX B}

\section{ASYMPTOTIC BEHAVIOR OF ODES}

Consider the ODE:

$$
\dot{\theta}=F(\theta),
$$

which we assume to have a unique solution for each initial condition defined on $\mathbb{R}^{+}$. We write $\Phi(t, \theta(0))$ the value at $t$ of the solution for initial condition $\theta(0)$. We denote by $d_{\mathcal{U}}(\theta)=\inf _{u \in \mathcal{U}}\|\theta-u\|$ the distance to set $\mathcal{U}$. We say that $\mathcal{U}$ is invarient if $\theta(0) \in \mathcal{U}$ implies $\Phi(t, \theta(0)) \in \mathcal{U}$ , $t \in \mathbb{R}^{+}$. We say that $\mathcal{U}$ is Lyapunov stable if for all $\delta_{1}>0$ there exists $\delta_{2}>0$ such that $d_{\mathcal{U}}(\theta(0)) \leq \delta_{2}$ implies $d_{\mathcal{U}}(\Phi(t, \theta(0))) \leq \delta_{1}, t \in \mathbb{R}^{+}$. A compact invariant set $\mathcal{U}$ is an attractor if there is an open invariant set $\mathcal{A}$ such that $\theta(0) \in \mathcal{A}$ implies $d_{\mathcal{U}}(\Phi(t, \theta(0))) \underset{t \rightarrow+\infty}{\rightarrow} 0 . \mathcal{A}$ is called the basin of attraction. A compact invariant set $\mathcal{U}$ is locally asymptotically stable if it is both Lyapunov stable and an attractor. If its basin of attraction $\mathcal{A}$ is equal to the whole space then $\mathcal{U}$ is globally asymptotically stable. Asymptotic stability is often characterized using Lyapunov functions. A 
positive, differentiable function $V: \mathbb{R}^{I} \rightarrow \mathbb{R}$, is said to be a Lyapunov function if $t \mapsto V(\Phi(t, \theta(0)))$ is decreasing, and strictly decreasing whenever $V(\Phi(t, \theta(0)))>0$. Then the set of zeros of $V$ is locally asymptotically stable. If we add the condition $V(\theta) \underset{\|\theta\| \rightarrow+\infty}{\rightarrow}+\infty$, then we have global asymptotic stability.

\section{APPENDIX C LINEAR ODES}

Consider the ODE:

$$
\dot{\theta}=A\left(\theta-\theta^{*}\right) .
$$

Its solution has the form:

$$
\theta(t)=\theta^{*}+e^{t A}\left(\theta(0)-\theta^{*}\right) .
$$

We denote by $\prec$ positive negativity for symmetric matrices. $\theta^{*}$ is asymptotically stable iff all the eigenvalues of $A$ have a strictly negative real part. Alternatively, asymptotic stability applies iff there exists $0 \prec X$ such that $A^{T} X+X A \prec 0$. In this case, $V(\theta)=\left(\theta-\theta^{*}\right)^{T} X\left(\theta-\theta^{*}\right)$ is a Lyapunov function for the ODE. The reader can refer to [28] for the linear matrix inequality approach to stability.

\section{APPENDIX D}

\section{DEFINITION OF DIAGONAL STRICT CONCAVITY}

Diagonal strict concavity is a property introduced in [14] for analyzing equilibrium of n-person games. Consider $I$ functions $\theta \rightarrow g_{i}(\theta)$ defined on a convex closed bounded set $S \subset \mathbb{R}^{I}$, and $w_{i}, i=1, \ldots, I$ some real positive constants. And denote by

$$
J G(\theta)=\left[\begin{array}{c}
w_{1} \nabla_{1} g_{1}(\theta) \\
\cdot \\
\cdot \\
w_{I} \nabla_{I} g_{I}(\theta)
\end{array}\right] .
$$

We say that $G(\theta)=\sum_{i=1}^{I} w_{i} g_{i}(\theta)$ is diagonally strictly concave for $\theta \in S$ if for every $\theta_{0}, \theta_{1} \in S$ we have

$$
\left(\theta_{0}-\theta_{1}\right)^{T} J G\left(\theta_{0}\right)+\left(\theta_{0}-\theta_{1}\right)^{T} J G\left(\theta_{1}\right)>0
$$

\section{APPENDIX E MARTINGALES}

Martingales are commonly used to characterize noise in stochastic approximation algorithms [18], [21]. We hereby give a succinct definition of martingales and martingale differences along with an insight in why they are useful.

Let $(\Omega, \mathcal{F}, \mathcal{P})$ denote a probability space, where $\Omega$ is the sample space, $\mathcal{F}$ a $\sigma$-algebra of subsets of $\Omega$, and $P$ a probability measure on $(\Omega, \mathcal{F})$. Let $\left\{M_{n}\right\}$ be a sequence of realvalued random variables defined on $(\Omega, \mathcal{F})$. If $\mathbb{E}\left(\left|M_{n}\right|\right)<\infty$ and

$$
\mathbb{E}\left(M_{n+1} \mid M_{i}, i \leq n\right)=M_{n}
$$

then $\left\{M_{n}\right\}$ is a martingale sequence. In this case, the sequence $N_{n}=M_{n}-M_{n-1}$ is a martingale difference sequence.
An important result on martingales is the martingale convergence theorem which proves that martingale sequences converge with probability 1 . This result is useful to characterize convergence of SA algorithms which model the noise as martingale differences (see [21] and [18]).

\section{REFERENCES}

[1] W. Luo, J. Zeng, X. Su, J. Li, and L. Xiao, "A mathematical model for joint optimization of coverage and capacity in self-organizing network in centralized manner," in 7th International ICST Conference on Communications and Networking in China (CHINACOM), 2012, pp. 622-626.

[2] J. Suga, Y. Kojima, and M. Okuda, "Centralized mobility load balancing scheme in lte systems," in 8th International Symposium on Wireless Communication Systems (ISWCS), 2011, pp. 306-310.

[3] R. Combes, Z. Altman, and E. Altman, "Self-organization in wireless networks: a flow-level perspective," in Proceedings of IEEE INFOCOM, 2012.

[4] —, "Interference coordination in wireless networks: a flow-level perspective," in Proceedings of IEEE INFOCOM, 2013.

[5] T. Jansen, M. Amirijoo, U. Turke, L. Jorguseski, K. Zetterberg, R. Nascimento, L. Schmelz, J. Turk, and I. Balan, "Embedding multiple self-organisation functionalities in future radio access networks," in IEEE 69th Vehicular Technology Conference (VTC Spring 2009), 2009, pp. 1-5.

[6] L.-C. Schmelz, M. Amirijoo, A. Eisenblaetter, R. Litjens, M. Neuland, and J. Turk, "A coordination framework for self-organisation in LTE networks," in IFIP/IEEE International Symposium on Integrated Network Management (IM). IEEE, 2011, pp. 193-200.

[7] A. Galani, K. Tsagkaris, P. Demestichas, G. Nguengang, I. BenYahia, M. Stamatelatos, E. Kosmatos, A. Kaloxylos, and L. Ciavaglia, "Core functional and network empower mechanisms of an operator-driven, framework for unifying autonomic network and service management," in IEEE 17th International Workshop on Computer Aided Modeling and Design of Communication Links and Networks (CAMAD), 2012, pp. 191-195.

[8] Z. Liu, P. Hong, K. Xue, and M. Peng, "Conflict avoidance between mobility robustness optimization and mobility load balancing," in IEEE Global Telecommunications Conference (GLOBECOM 2010), 2010, pp. $1-5$.

[9] H. Klessig, A. Fehske, G. Fettweis, and J. Voigt, "Improving coverage and load conditions through joint adaptation of antenna tilts and cell selection rules in mobile networks," in IEEE International Symposium on Wireless Communication Systems (ISWCS), 2012, pp. 21-25.

[10] R. Combes, Z. Altman, and E. Altman, "Self-organizing relays: dimensioning, self-optimization, and learning," IEEE Transactions on Network and Service Management, vol. 9, Dec. 2012.

[11] P. Vlacheas, E. Thomatos, K. Tsagkaris, and P. Demestichas, "Operatorgoverned son coordination in downlink LTE networks," in Future Network Mobile Summit (FutureNetw), 2012, pp. 1-9.

[12] 3GPP, "Self-Organizing Networks (SON) Policy Network Resource Model (NRM) Integration Reference Point (IRP); Information Service (IS)," 3rd Generation Partnership Project (3GPP), TS 28.628, June 2013.

[13] R. Combes, Z. Altman, and E. Altman, "Coordination of autonomic functionalities in communications networks," in Proceedings of the 8th International Symposium on Modeling and Optimization in Mobile, Ad Hoc and Wireless Networks (WiOpt), 2013.

[14] J. B. Rosen, "Existence and Uniqueness of Equilibrium Points for Concave N-Person Games," Econometrica, vol. 33, no. 3, pp. 520-534, 1965.

[15] P. Hartman, "A lemma in the theory of structural stability of differential equations," Proceeding of the American Mathematical Society, vol. 11, pp. 610-620, 1960. 
[16] R. Björck, Numerical methods for least squares problems. SIAM, 1996.

[17] D. P. Bertsekas and J. N. Tsitsiklis, Parallel and distributed computation: numerical methods. Upper Saddle River, NJ, USA: Prentice-Hall, Inc., 1989.

[18] H. J. Kushner and G. G. Yin, Stochastic Approximation and Recursive Algorithms and Applications 2nd edition. Springer Stochastic Modeling and Applied Probability, 2003.

[19] J.-H. Yun and K. G. Shin, "Ctrl: a self-organizing femtocell management architecture for co-channel deployment," in Proceedings of the sixteenth annual international conference on Mobile computing and networking. ACM, 2010, pp. 61-72.

[20] I. Viering, M. Dottling, and A. Lobinger, "A mathematical perspective of self-optimizing wireless networks," in Communications, 2009. ICC'09. IEEE International Conference on. IEEE, 2009, pp. 1-6.

[21] V. S. Borkar, Stochastic Approximation: A Dynamical Systems Viewpoint. Cambridge University Press, 2008.

[22] F. Gantmacher, Applications Of The Theory Of Matrices, ser. Dover Books on Mathematics Series. Dover, 2005.

[23] M. E. Fisher and A. T. Fuller, "On the stabilization of matrices and the convergence of linear iterative processes," Mathematical Proceedings of the Cambridge Philosophical Society, vol. 54, pp. 417-425, 101958.

[24] C. P. Simon and L. Blume, Mathematics for economists. Norton New York, 1994, vol. 7.

[25] S. Roy, J. Minteer, and A. Saberi, "Some new results on stabilization by scaling," in American Control Conference, 2006, 2006, pp. 6 pp.-.

[26] A. Pyzara, B. Bylina, and J. Bylina, "The influence of a matrix condition number on iterative methods' convergence," in IEEE Federated Conference on Computer Science and Information Systems (FedCSIS). IEEE, 2011, pp. 459-464.

[27] T. Huckle and A. Kallischko, "Frobenius norm minimization and probing for preconditioning," International Journal of Computer Mathematics, vol. 84, no. 8, pp. 1225-1248, 2007.

[28] S. Boyd, L. El Ghaoui, E. Feron, and V. Balakrishnan, Linear Matrix Inequalities in System and Control Theory, ser. Studies in Applied Mathematics. Philadelphia, PA: SIAM, Jun. 1994, vol. 15. 